\title{
Doping and Field-Induced Insulator-Metal Transitions in Half-Doped Manganites
}

\author{
O. Cépas, ${ }^{1,2}$ H. R. Krishnamurthy, ${ }^{1,3}$ and T. V. Ramakrishnan ${ }^{1,3,4}$ \\ ${ }^{1}$ Centre for Condensed Matter Theory, Department of Physics, Indian Institute of Science, Bangalore 560012, India \\ ${ }^{2}$ Institut Laue Langevin, BP 156, 38042 Grenoble, France \\ ${ }^{3}$ Jawaharlal Nehru Centre for Advanced Scientific Research, Jakkur, Bangalore 560 064, India \\ ${ }^{4}$ Department of Physics, Banaras Hindu University, Varanasi 221005, India
}

\begin{abstract}
We argue that many properties of the half-doped manganites may be understood in terms of a new two( $e_{g}$ electron)-fluid description, which is energetically favorable at intermediate Jahn-Teller (JT) coupling. This emerges from a competition between canting of the core spins of Mn promoting mobile carriers and polaronic trapping of carriers by JT defects, in the presence of magnetic (CE), orbital and charge order. Using it we explain features of the doping and magnetic-field-induced insulator-metal transitions such as the electron-hole asymmetry and the smallness of the transition fields.
\end{abstract}

"Half-doped" manganites such as $R_{1-x} \mathrm{~A}_{x} \mathrm{MnO}_{3}$ with $x=1 / 2$ where $R$ is a $3+$ rare-earth ion and $\mathrm{A}$ a $2+$ alkaline earth ion have been the object of extensive studies for many years [1]. The magnetic order in the lowest temperature phase is either CE-type [2], i.e., ferromagnetic zig-zag chains with relative antiferromagnetic (AFM) order (as in $\mathrm{La}_{1 / 2} \mathrm{Ca}_{1 / 2} \mathrm{MnO}_{3}$, where it was first proposed [2,3], $\mathrm{Nd}_{1 / 2} \mathrm{Sr}_{1 / 2} \mathrm{MnO}_{3}$ [4], and $\mathrm{Nd}_{1 / 2} \mathrm{Ca}_{1 / 2} \mathrm{MnO}_{3}$ [5]) or A-type, i.e., ferromagnetic planes with relative AFM alignment (as in $\operatorname{Pr}_{1 / 2} \mathrm{Sr}_{1 / 2} \mathrm{MnO}_{3}$ [4]). The competition between the $\mathrm{CE}$ and $\mathrm{A}$ phases appears even in a simple one-orbital model [6] because of the interplay of ferromagnetic double exchange and AFM superexchange between the core $t_{2 g}$ spins of $\mathrm{Mn}$ (see also Fig. 1). However, the $\mathrm{Mn}^{3+} / \mathrm{Mn}^{4+}$ charge order $(\mathrm{CO})$ and alternating $\left(3 x^{2}-r^{2}\right) /\left(3 y^{2}-r^{2}\right)$ orbital order (OO) proposed for the CE phase [3] is controversial [7-9]. X-ray diffraction data suggests sizeable Jahn-Teller (JT) distortions $[4,10]$ with two inequivalent Mn sites, with valence $3.5 \pm \delta$, but $\delta$ is hard to determine. A realistic two Mn- $e_{g}$ orbital model [11] does lead to OO, and the inclusion of Coulomb [11-13], and JT [14,15] interactions to the correct charge stacked $\mathrm{CO}$, with $\delta \lesssim$ 0.2 being model parameter dependent. However, neutron diffraction data in $\mathrm{Pr}_{0.6} \mathrm{Ca}_{0.4} \mathrm{MnO}_{3}$ has been interpreted in terms of "Zener polarons" with $\delta \ll 1$ [8], though this is not confirmed in $\operatorname{Pr}_{0.5} \mathrm{Ca}_{0.5} \mathrm{MnO}_{3}$ [16], and could require the added electrons [17]. An alternate, closely related picture is that of a bond-charge-density wave, with no charge contrast of the Mn ions, substantial hole occupancy on the oxygen ions on the chains, and alternating " $\mathrm{O}^{2-} / \mathrm{O}^{-}$" order $[17,18]$.

Apart from these, other fundamental issues remain to be understood. One is the striking asymmetry with respect to the addition of electrons or holes. Experimentally, added electrons typically favor ferromagnetic metallic phases while added holes favor insulating phases [1]. In contrast, band-structure arguments [11], and treatments including adiabatic JT distortions [15] lead to metallic phases on both sides. Another puzzling but ubiquitous feature [1] first seen in $(\mathrm{Nd}, \mathrm{Sm})_{1 / 2} \mathrm{Sr}_{1 / 2} \mathrm{MnO}_{3}$ [19] is that magnetic-fields ( 10-40 Tesla) much smaller than the Néel or charge ordering temperatures $(\sim 200 \mathrm{~K})$ induce an insulatormetal transition. An explanation is that this arises from the proximity of the systems to the CE-FM phase boundary $[6,13,20]$; but this requires a fine tuning of parameters which is difficult to justify.

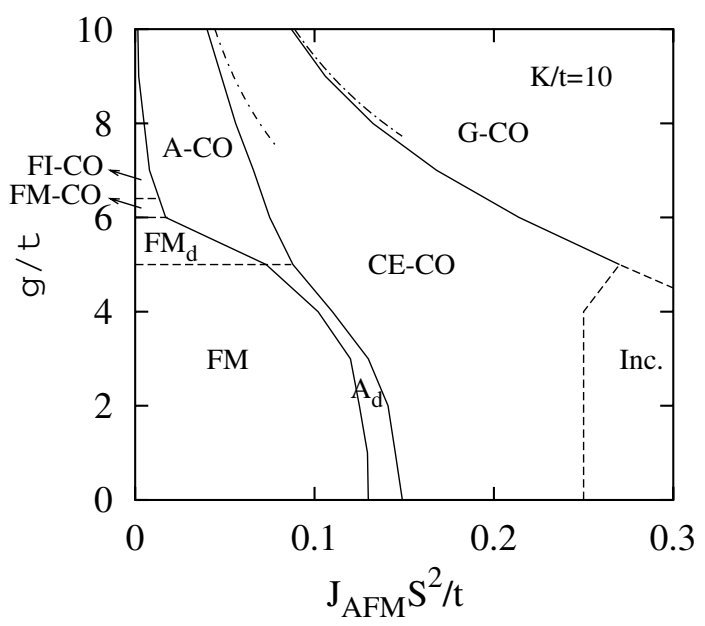

FIG. 1. Phase diagram of the 3D two-orbital model $(T=0$, $x=0.5, K / t=10)$. FM $\left(\mathrm{FM}_{d}\right)$ : ferromagnetic metallic phase with no distortions (small uniform distortions). FI-CO (FM$\mathrm{CO}$ ): charge-ordered ferromagnetic insulating (metallic) phase with distortions that favor occupancy of the $x^{2}-y^{2}$ orbitals. $\mathrm{A}_{d}$ : ferromagnetic planes AFM aligned with uniform distortions. A-CO: A with charge order. CE-CO: Ferromagnetic zig-zag chains AFM ordered, orbital ordered $\left(3 x^{2}-r^{2} / 3 y^{2}-r^{2}\right)$, and charge-ordered. G-CO: Néel AFM phase with charge-order. Inc.: Possible incommensurate states that interpolate between $\mathrm{CE}$ and G. Dotted dashed lines come from analytical expressions derived in the strong-coupling limit. Solid (dashed) lines show first-order (second-order) phase transitions. 
Recently, starting from a large JT coupling picture, a two-fluid $e_{g}$ electron model, one polaronic and localized, and the other bandlike and mobile, was proposed and shown to explain many properties of manganites in the orbital liquid regime [21]. In this Letter, we show how a similar picture emerges from a realistic microscopic model, even at intermediate JT couplings and in the halfdoped case (i.e., in the presence of orbital and charge order), due to a competition between the promotion of mobile carriers by the canting of Mn core spins, and the polaronic trapping of carriers due to the JT coupling. This leads to natural explanations for the particle-hole asymmetry around half-doping as well as the magnetic-fieldinduced insulator-metal transition at half-doping mentioned above. Interestingly, a similar two-carrier-type hypothesis was proposed based on phenomenological grounds to understand resistivity data in $\mathrm{La}_{1-x} \mathrm{Ca}_{x} \mathrm{MnO}_{3}$ $(x \sim 1 / 2)$ [22]. More recently, a particle-hole asymmetric Ginzburg-Landau theory with competing ferromagnetic and charge order was used to explain [23] the incommensurate charge order [24] seen for $x>0.5$. Our theory provides a microscopic basis for both of the above. JT distortions were recently tracked as function of field in $\mathrm{La}_{1 / 2} \mathrm{Ca}_{1 / 2} \mathrm{MnO}_{3}$ and shown to play a crucial role near the field-induced transition [25,26]; our picture is completely consistent with this. The ideas presented here may be relevant to other classes of systems such as $\mathrm{CsC}_{60}$, in which a similar two-electron phase has been proposed [27].

Our theory is based on the following microscopic, twoorbital Hamiltonian for the manganites:

$$
\begin{aligned}
\mathcal{H}\left[\left\{\mathbf{S}_{i a}, Q_{i a}, \Theta_{i a}\right\}\right]= & -\sum_{i j \alpha \beta a b} \tilde{t}_{a b i j}^{\alpha \beta}\left(\mathbf{S}_{i a}, \mathbf{S}_{j b}\right) c_{i a \alpha}^{\dagger} c_{j b \beta} \\
& +\sum_{\langle i a, j b\rangle} J_{\mathrm{AF}} \mathbf{S}_{i a} \cdot \mathbf{S}_{j b} \\
& -g \mu_{B} \sum_{i a} \mathbf{H} \cdot \mathbf{S}_{i a}+\frac{1}{2} K \sum_{i a} Q_{i a}^{2} \\
& -g \sum_{i a \alpha \beta} Q_{i a} c_{i a \alpha}^{\dagger} \tau_{\alpha \beta}\left(\Theta_{i a}\right) c_{i a \beta} .
\end{aligned}
$$

Here $c_{i a \alpha}^{\dagger}$ (with $\alpha=x^{2}-y^{2}, 3 z^{2}-r^{2}$ ) creates an electron in an effective, "low-energy-projected" Wannier orbital with $e_{g}$ symmetry in the unit cell $i$ and a sublattice site $a$. (We use a 8-sublattice decomposition to accommodate the CE phase.) There are $N$ sites and $c N$ electrons with $c \equiv$ $(1-x) \simeq 1 / 2$. Because of a large Hund's coupling $J_{\mathrm{H}}$ the electron spin is assumed to be locked parallel to the $S=$ $3 / 2 t_{2 g}$ core spins of Mn, modeled as classical vectors $\mathbf{S}_{i a}$. The hopping parameters (with $4 t / 3$ being the hopping between $\left(3 z^{2}-r^{2}\right)$ orbitals in the $z$ direction) include the standard Anderson-Hasegawa dependence on $\mathbf{S}_{i a}, \mathbf{S}_{j b}$ due to the $J_{\mathrm{H}} \rightarrow \infty$ projection [15]. The core spins are directly coupled by an AFM superexchange, $J_{\mathrm{AFM}} S^{2} \sim 0.1 t$ [15]. $\mathbf{H}$ is the external magnetic field. The last two terms include the vibrational energy of JT phonons (where $K$ is the lattice stiffness of a simplified non-cooperative model) and their coupling, $g$, to the $e_{g}$ electrons. $Q_{i a}$ and $\Theta_{i a}$ represent the amplitude and the angle of the two $\left(Q_{2}, Q_{3}\right) \mathrm{JT}$ modes, and the $\tau$ matrix the symmetry of their coupling [15]. Our neglect of on-site Coulomb interactions is reasonable because large JT distortions and large $J_{H}$ by themselves suppress double occupancy; and of long-range Coulomb interactions, breathing modes, and cooperative JT couplings, because these will lead to quantitative and not qualitative differences [28].

We first determine the ground state of (1) exactly numerically, by minimizing the electronic energy with respect to spin and JT distortion variables but in a subspace restricted to be periodic with a unit cell of at most 8 sites. This accommodates the CE state as well as several other competing commensurate states. Compared to earlier numerical approaches [15] that were limited to small clusters, our calculations are practically in the thermodynamic limit. Our phase diagram, given in Fig. 1 (with phases described in the caption) is consistent with previous results $[15,20]$. In addition, we have detailed predictions on $Q_{i a}^{(0)}$, the strength of the JT distortions in the ground state, etc. [28]. The strong-coupling phases, all insulating and charge ordered, can be understood by starting from electrons trapped in Wannier orbitals on alternate JT distorted sites. Virtual double exchange [21], an exchange mechanism [of order $t^{2} / E_{\mathrm{JT}}$ where $E_{\mathrm{JT}}=g^{2} /(2 K)$ is the JT binding energy] arising from virtual hopping of these electrons to neighboring empty sites with aligned core spins and dependent upon the orientation of the JT distortion or occupied orbital [28], competes with $J_{\mathrm{AFM}}$ in determining the phases. A comparison of the energies of the various phases leads to the sequence of first-order transitions at couplings given by $J_{\mathrm{AFM}} S^{2}=4 t K /\left(9 g^{2}\right)$ and $J_{\mathrm{AFM}} S^{2}=8 t K /\left(9 g^{2}\right)$ (dotted dashed lines in Fig. 1).

We next study the instabilities of the homogeneous insulating phases discussed above with respect to particle or hole excitations accompanied by single site defects in their JT distortion pattern. For this, we find the electronic eigenvalues of (1) in the presence of such defects numerically (for $N$ up to 1728), and calculate the energy cost or gain from filling the energy levels with $c N$ electrons.

To start with, consider the ferromagnetic insulator (FI)$\mathrm{CO}$ phase, with the electrons localized at alternate JT distorted sites with distortion $Q^{(0)}(\simeq g / K$ at strong coupling, obtained in general by minimization as discussed above). Consider promoting an electron across the charge gap. We find that it is energetically favorable for the hole to localize at a site by relaxing the local JT distortion to $Q^{(0)}-Q_{d}$. The loss in electronic energy is overcompensated by the gain in elastic energy. Such mixed excitations, with the hole polaronically trapped while the electron is mobile, thus have lower energies than mobile particle-hole excitations of the ground state band structure. This is demonstrated in Fig. 2 where, in addition to the minimum at $Q_{d}=0$ (corresponding to the homogeneous phase), there is another minimum at $Q_{d} \sim Q^{(0)}$, corresponding to 
the removal of the JT distortion at one site. Furthermore, this minimum becomes an instability for $g / t \lesssim 6.8$ (Fig. 2), although the other homogeneous phases of Fig. 1 are at higher energy at this $g / t$, suggesting that there might be an inhomogeneous phase where such defects proliferate. With a small number of such defects, a small fraction of electrons are converted from localized to mobile states leading to a metal with a small concentration of mobile electrons, while the majority of the electrons are still polaronically trapped at JT distorted sites. Thus we are led to the two-fluid picture [21] again, but now extended to include orbital and charge order [28].

We next show that similar instabilities arise in the CE phase doped with carriers, and underlie the strong asymmetry between hole and electron doping seen experimentally. For this purpose, we include the effects of canting promoted by the addition of carriers [29] and obtain the optimal (i.e., energy minimizing) canting angle as a function of $x$ close to $1 / 2$. But, similarly to the case of the FI$\mathrm{CO}$ phase discussed above, the homogeneous canted phases are in competition with inhomogeneous phases where the added carriers are self-trapped by JT distortions if $g / t$ is sufficiently large. The resulting "phase" diagram is shown in Fig. 3. On the electron-doped side $(x<1 / 2)$, it is favorable to trap the added electrons for small $\delta c \equiv$ $1 / 2-x$. leading to an insulating uncanted CE phase (denoted CE-T in Fig. 3). Increasing $\delta c$ leads eventually to a canted metallic phase (CE-M-C) via a first-order transition, as the JTenergy gain due to trapping is linear in $\delta c, \delta E_{t r}=$ $-\tilde{E}_{\mathrm{JT}}^{e}|\delta c|$ (with $\tilde{E}_{\mathrm{JT}}^{e}$ obtained by solving the one-defect problem mentioned above with one added electron) [28], whereas the energy gain from canting is quadratic, $\delta E_{c a} \sim$ $-(\delta c)^{2}$ [29]. The latter loses for small $\delta c$ but wins for larger $\delta c$. On the hole side, however, we find that canted phases are never energetically favorable. The asymmetry arises because of the nature of the CE ordering. Canting

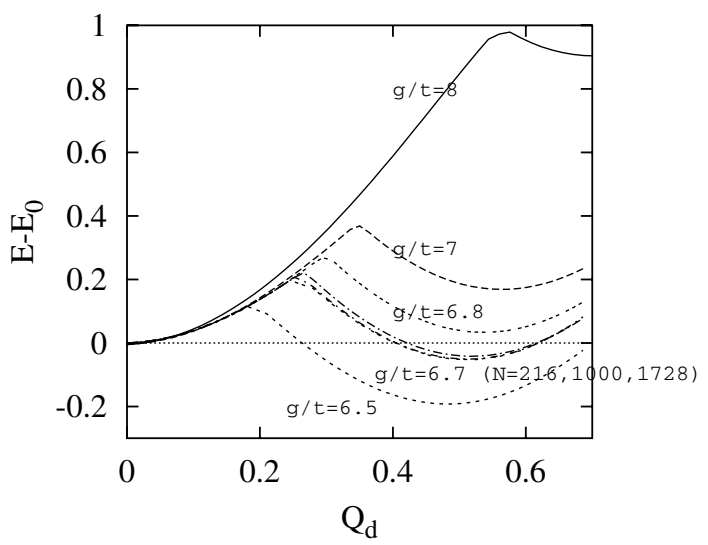

FIG. 2. Energy change when a single JT defect is introduced in the FI-CO phase. $Q^{(0)}-Q_{d}$ is the JT distortion on a defect site; all the other occupied sites having the same distortion $Q^{(0)}$. The softening of the excitation with $Q_{d} \sim Q^{(0)}$ at $g / t \sim 6.8$ signals a phase transition with proliferation of defects. Finite-size effects are small and shown for $g / t=6.7$. leads to a 2D dispersion, with a large density of states at the bottom of the conduction band (where doped electrons go), whereas it gives a 3D dispersion, with a vanishing density of states at the top of the valence band (where doped holes go). Hence canting angles get large when electrons are added and compete effectively against electron trapping. But when holes are added, canting is never competitive. The holes get trapped by JT distortions for $g / t \gtrsim 4$ and the system is insulating. Thus, our approach leads to an explanation for the asymmetry between particle and hole doping seen experimentally, and the appearance of incommensurate charge-ordered phases on the hole doped side [23,24,28]. On the electron-doped side, other phases could appear [17,21] at finite doping, but one needs to go beyond the instability calculations reported here to address these.

An external magnetic-field applied to the CE phase also promotes canting. Experimentally, as discussed earlier, a field-induced insulator-metal transition occurs at extremely small fields. To locate the transition in our theory, we minimize and compare the energies of various 8sublattice structures in a field, including the JT-distorted canted CE (D-C-CE) state, the undistorted canted (U-C) state with the optimal (high) canting angle, etc., We find that for $g / t<5$ the ground state switches in a first-order transition from a D-C-CE phase (with the canting hardly changing the JT distortions) to an U-C (or FM) phase with increasing field. At the transition, the system becomes metallic, the magnetization jumps (Fig. 4), and the JT distortions relax to zero in agreement with recent experiments $[25,26]$. The transition fields depend strongly on $g / t$ as is clear from Fig. 4. For $g / t \gtrsim 6.8$, the FM state is insulating and no insulator-metal transition can be found, indicating that $5.0 \leqq g / t \lesssim 6.8$ for manganites (we need $g / t \gtrsim 5.0$ to obtain the A-CO phase [4] [see Fig. 1]). This is just the range of $g / t$ where JT-defect instabilities of the

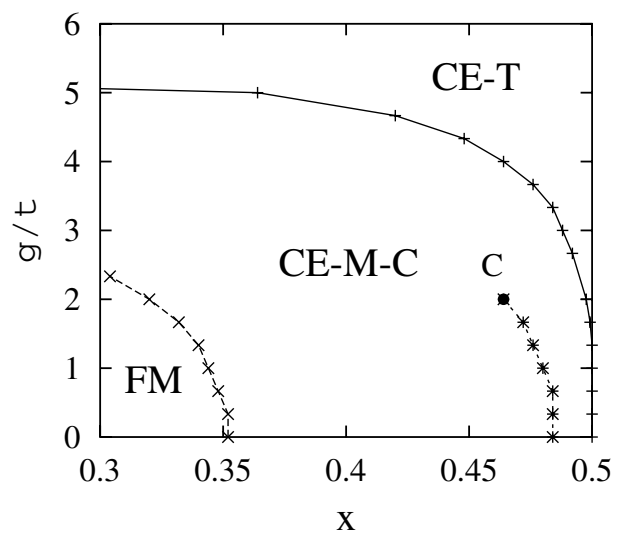

FIG. 3. Phase diagram, $g / t$ vs doping, $x\left(J_{\mathrm{AFM}} S^{2} / t=0.15\right)$. CE-M-C: canted CE state with distortions and charge-order (metallic). CE-T: CE phase with extra carriers trapped in JT distortions (insulating). FM: ferromagnetic metallic phase with no distortions. The upper curve is valid for $x$ close enough to 0.5 . $\mathrm{C}$ is a critical point ending a first-order line between two canted states (with different canting angles). 


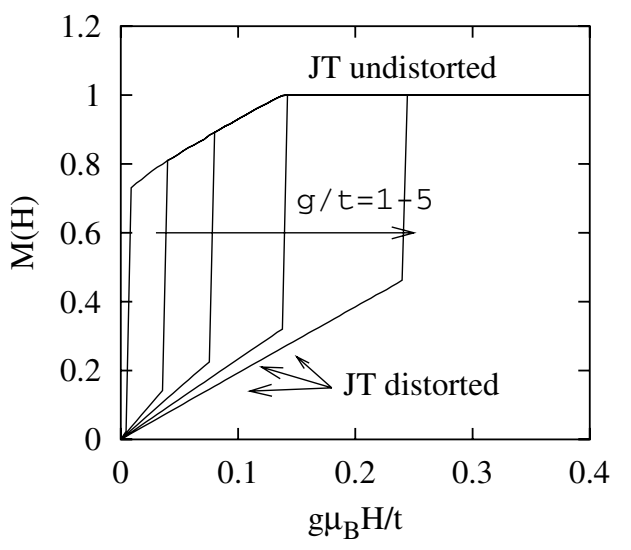

FIG. 4. Magnetization vs field $\left(g / t=1-5, \quad J_{\mathrm{AFM}} S^{2} / t=\right.$ $0.15)$. The first-order transition to an undistorted highly canted (or fully FM) metallic phase is accompanied with a relaxation of the JT distortions.

sort discussed above arise, which suggests that the fieldinduced metallic phase needs a 2-fluid description.

However, the transition fields obtained in our calculations are too large compared to experiments. For instance, $g \mu_{B} H_{c} \sim 0.1 t$ (Fig. 4), gives $H_{c} \sim 140 \mathrm{~T}$ (with $t \sim$ $0.2 \mathrm{eV})$. The discrepancy is connected with the overestimation of the charge gap in our model, due to the neglect of (1) the finiteness of the Hund's coupling, which would allow for hopping even between sites with antialigned core spins; (2) the cooperative nature of the JT phonons, causing sizeable distortions on the corner sites; and (3) the small second neighbor hopping. Indeed, if we estimate the transition field using the experimentally observed charge gap, we get numbers in reasonable agreement with observations.

In conclusion, our work indicates that the competition between canting (i.e., not full ferromagnetism, which could be checked by neutron diffraction) induced metallicity and inhomogeneity arising from the trapping of carriers by JT defects is responsible for several features of half-doped manganites, e.g., the particle-hole asymmetry in doping, and the doping or field-induced insulator-metal transitions. This leads to a new two-fluid model with localized and mobile electrons, which extends the work of Ref. [21] to include orbital and charge order, which when treated with more sophisticated methods such as dynamical mean-field theory could yield a satisfactory and complete theory of doped manganites including the regime near half-doping.

O. C. would like to thank G. Bouzerar, T. Chatterji, G. Jackeli, D. Khomskii, Y. Motome, H. Nojiri, and T. Ziman for stimulating discussions. O.C. and H.R.K. acknowledge financial support from IFCPAR, Grant No. 2404-1.

[1] Colossal Magnetoresistance Oxides, edited by Y. Tokura (Gordon and Breach, New York, 2000); M. B. Salamon and M. Jaime, Rev. Mod. Phys. 73, 583 (2001); E.
Dagotto, T. Hotta, and A. Moreo, Phys. Rep. 344, 1 (2001).

[2] E. O. Wollan and W. C. Koehler, Phys. Rev. 100, 545 (1955).

[3] J. B. Goodenough, Phys. Rev. 100, 564 (1955).

[4] H. Kawano et al., Phys. Rev. Lett. 78, 4253 (1997); H. Kawano-Furukawa et al., Phys. Rev. B 67, 174422 (2003).

[5] F. Millange, S. de Brion, and G. Chouteau, Phys. Rev. B 62, 5619 (2000).

[6] S. K. Mishra, R. Pandit, and S. Satpathy, Phys. Rev. B 56, 2316 (1997).

[7] J. Garcia et al., J. Phys. Condens. Matter 13, 3229 (2001).

[8] A. Daoud-Aladine et al., Phys. Rev. Lett. 89, 097205 (2002).

[9] M. Coey, Nature (London) 430, 155 (2004).

[10] P. G. Radaelli et al., Phys. Rev. Lett. 75, 4488 (1995); Phys. Rev. B 55, 3015 (1997).

[11] J. van den Brink, G. Khaliullin, and D. Khomskii, Phys. Rev. Lett. 83, 5118 (1999).

[12] G. Jackeli, N. B. Perkins, and N. M. Plakida, Phys. Rev. B 62, 372 (2000).

[13] S. Fratini, D. Feinberg, and M. Grilli, Eur. Phys. J. B 22, 157 (2001).

[14] Z. Popovic and S. Satpathy, Phys. Rev. Lett. 88, 197201 (2002).

[15] T. Hotta, A.-L. Malvezzi, and E. Dagotto, Phys. Rev. B 62, 9432 (2000); S. Yunoki, T. Hotta, and E. Dagotto, Phys. Rev. Lett. 84, 3714 (2000).

[16] R. J. Goff and J.P. Attfield, Phys. Rev. B 70, 140404R (2004).

[17] D. Efremov, J. van den Brink, and D. Khomskii, Nat. Mater. 3, 853 (2004).

[18] G. Zheng and C. H. Patterson, Phys. Rev. B 67, 220404R (2003); V. Ferrari, M. Towler, and P. B. Littlewood, Phys. Rev. Lett. 91, 227202 (2003).

[19] Y. Tomioka et al., Phys. Rev. Lett. 74, 5108 (1995); Y. Tokura et al., ibid. 76, 3184 (1996); Y. Tokura and Y. Tomioka, J. Magn. Magn. Mater. 200, 1 (1999).

[20] H. Aliaga et al., Phys. Rev. B 68, 104405 (2003).

[21] T. V. Ramakrishnan et al., Phys. Rev. Lett. 92, 157203 (2004); See also cond-mat/0308396.

[22] M. Roy et al., Phys. Rev. B 58, 5185 (1998).

[23] G.C. Milward, M.J. Calderon, and P.B. Littlewood, Nature (London) 433, 607 (2005).

[24] S. Mori, C. H. Chen, and S.-W. Cheong, Nature (London) 392, 473 (1998); J. C. Loudon et al., Phys. Rev. Lett. 94, 097202 (2005); L. Brey, Phys. Rev. Lett. 92, 127202 (2004).

[25] T. A. Tyson et al., Phys. Rev. B 70, 024410 (2004).

[26] H. Nojiri (private communication).

[27] V. Brouet et al., Phys. Rev. Lett. 82, 2131 (1999); Phys. Rev. B 66, 155123 (2002).

[28] The details will be published elsewhere. The charge contrasts we obtain, e.g., $\delta \lesssim 0.2$ for $g / t=5$, are far from the ionic picture $\delta=0.5$ and can get reduced further with the inclusion of cooperative JT effects, etc. The distribution of the charge on the $\mathrm{Mn}$ and $\mathrm{O}$ ions in the chains would be governed by the precise composition of the Wannier orbital, and could interpolate between the various conflicting pictures of $\mathrm{CO}$ mentioned earlier depending on the details of the band-structure parameters.

[29] P.-G. de Gennes, Phys. Rev. 118, 141 (1960). 